\title{
Microwave absorption properties of carbon based microwave absorber derived from chicken featherfiber
}

\author{
Hari Prakash ${ }^{1}$, Sandeep Kumar Singh ${ }^{1}$, M.J. Akhtar ${ }^{1,2}$ and Kamal K Kar ${ }^{1,3 *}$ \\ ${ }^{1}$ Department of Materials Science Programme, Indian Institute of Technology Kanpur, Kanpur- \\ 208016, India \\ ${ }^{2}$ Department of Electrical Engineering, Indian Institute of Technology Kanpur, Kanpur-208016, India \\ ${ }^{3}$ Department of Mechanical Engineering, Indian Institute of Technology Kanpur, Kanpur-208016, \\ India \\ *Email: kamalkk@iitk.ac.in
}

Sustainable growth of society and economic development are heavily dependent on waste management that offers minimum environmental hazard and maximum efficiency. Biomass derived carbon has been explored for making super capacitor and fuel cells $[1,2]$; but little effort was put to utilize these waste material for electromagnetic shielding and microwave absorption. Compared to current materials used in making of microwave absorbing materials, animal waste has a potential to be used as an alternate microwave absorbing materials at a very low cost and is environment friendly. In this proposed work, micro fibrous carbon has been synthesized by pyrolysis of chicken feather fiber (CFF) at different temperatures ranging between 400 and $700{ }^{\circ} \mathrm{C}$. The structural morphology was carried out using scanning electron microscopy (SEM). The complex permittivity spectra of the fabricated composites composed of CFF and epoxy were measured in the $\mathrm{X}$ band (8.2-12.4 GHz) frequency region. The complex permittivity results from $\mathrm{CFF} /$ Epoxy composites shows significant increase in permittivity as a function of temperature (Figure 1).
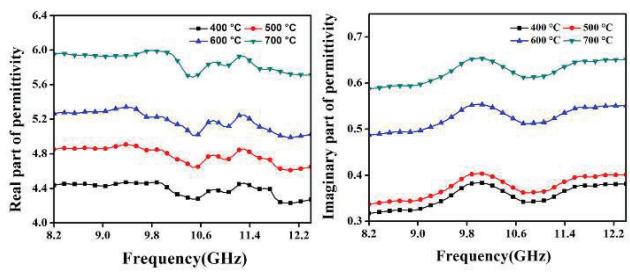

Figure 1: Complex permittivity of CFF (30 wt.\%)/epoxy composites at different pyrolysis temperature in X-band

The fabricated composites possess excellent absorption when CFF synthesized at
$\mathrm{T}_{\mathrm{p}} \geq 700{ }^{\circ} \mathrm{C}$; owing to high surface area and conductive nature of CFF filler. The CFF can be synthesized at higher temperature to exhibit substantial microwave absorption composite thickness of $2 \mathrm{~mm}$. The results from CFF/Epoxy composites shows significant increase in dielectric losses when temperature is increased and better performance is expected when pyrolysis temperature is increased further (Figure 2).

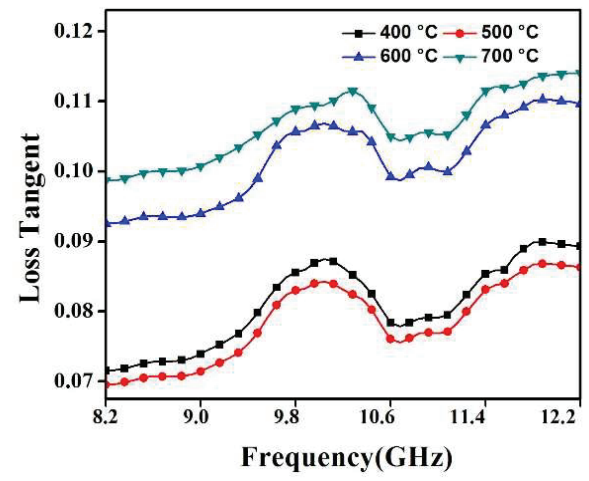

Figure 2: Frequency dependent loss tangent of CFF (30 wt.\%)/epoxy composites at different pyrolysis temperature in X-band

The aforementioned findings provide that $\mathrm{CFF}$ could be a potential filler candidate in the field of microwave absorber.

\section{References}

1. R. Sharma and K. K. Kar, Electrochimica Acta, 191, 2016, 876-886.

2. J. Briscoe, A. Marinovic, M. Sevilla, S. Dunn, M. Titirici, Biomass-Derived Carbon

3. Quantum Dot Sensitizers for Solid-State Nanostructured Solar Cells, Angew. Chem. Int. Ed. 54 (2015) 4463-4468. 